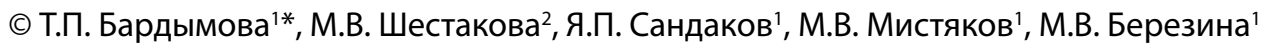

'Иркутская государственная медицинская академия последипломного образования - филиал Российской медицинской академии непрерывного профессионального образования, Иркутск, Россия ${ }^{2}$ Национальный медицинский исследовательский центр эндокринологии, Москва, Россия

ОБОСНОВАНИЕ. Структурно-метаболические нарушения костной ткани у женщин с сахарным диабетом 2 типа (СД2) чаще всего не имеют клинических проявлений, но сопровождаются риском переломов.

ЦЕЛЬ. Изучить показатели метаболизма костной ткани, микроархитектоники и минеральной плотности кости (МПК) у пациенток с СД2 бурятской популяции.

МАТЕРИАЛЫ И МЕТОДЫ. В наблюдательное одноцентровое одномоментное контролируемое исследование вошли 73 женщины, больные СД2, которых разделили на 2 группы в зависимости от функционального состояния яичников (репродуктивный или постменопаузальный периоды). В каждой группе были выделены подгруппы бурятской и русской популяции. В первую группу вошли 34 пациентки с СД2 репродуктивного периода: 16 бурятской популяции и 18 - русской популяции. Вторая группа состояла из 39 пациенток с СД2 постменопаузального периода: 17 - бурятской популяции и 22 - русской популяции. Проводилось исследование МПК в поясничном отделе позвоночника (L1-L4), шейке бедра (Neck), в проксимальном отделе бедренной кости (Total hip), трабекулярного костного индекса (ТКИ), остеокальцина (ОК), N-терминального пропептида проколлагена 1-го типа (P1NP), 25(OH) витамина D сыворотки крови, С-концевого телопептида коллагена I типа ( $\beta$-Cross laps) и ионизированного кальция плазмы крови (iCa).

РЕзУЛЬтАТЫ. У пациенток с СД2 репродуктивного периода бурятской популяции установлено повышение как маркеров остеосинтеза P1NP $(p=0,035)$, OK $(p=0,047)$, так и костной резорбции $\beta$-Cross laps $(p=0,040)$ относительно пациенток русской популяции. У женщин с СД2 в периоде постменопаузы бурятской популяции также наблюдалось повышение P1NP ( $p=0,016)$, OK $(p=0,048), \beta$-Cross laps $(p=0,020)$ сравнительно с женщинами в периоде постменопаузы русской популяции. Структурные нарушения, характеризующиеся снижением ТКИ, выявлены только в постменопаузальном периоде у пациенток бурятской популяции по сравнению с женщинами русской популяции $(p=0,029)$.

Сравнительный анализ у женщин с СД2 бурятской популяции в зависимости от функционального состояния яичников показал, что для женщин постменопаузального периода характерна активация костного ремоделирования с повышением P1NP ( $p=0,019)$, OK ( $p=0,004)$ и $\beta$-Cross laps $(p=0,004)$, сопровождающаяся снижением МПК Neck $(p=0,006)$, MПK Total hip $(p=0,003), M \Pi K L 1-L 4(p=0,049)$ и ТKИ $(p=0,020)$ относительно пациенток с СД2 в репродуктивном периоде.

ЗАКЛюЧЕНИЕ. У женщин бурятской популяции, больных СД2, как в репродуктивном, так и постменопаузальном периодах установлены повышение маркеров костного ремоделирования и стабильность МПК при сравнении с пациентками русской популяции. Постменопаузальный период характеризуется дополнительным снижением ТКИ у пациенток с СД2 бурятской популяции относительно женщин русской популяции.

КЛЮЧЕВЫЕ СЛОВА: сахарный диабет; остеосинтез; МПК; ТКИ; этнические особенности

\title{
ETHNIC CHARACTERISTICS OF BONE REMODELING IN FEMALE PATIENTS WITH TYPE 2 DIABETES MELLITUS
}

(c) Tatiana P. Bardymova1* , Marina V. Shestakova², Yakov P. Sandakov', Maksim V. Mistiakov' , Marina V. Berezina

'Irkutsk State Medical Academy of Postgraduate Education - Branch Campus of the Russian Medical Academy of Continuing Professional Education, Irkutsk, Russia

2Endocrinology Research Centre, Moscow, Russia

BACKGROUND: Structural and metabolic disorders of bone tissue in women with T2DM have no clinical manifestations, but they are accompanied by the risk of fractures.

AIM: To study the parameters of bone metabolism, BMD and microarchitectonics in female patients with T2DM in the Buryat population. 
MATERIALS AND METHODS: The observational single-center one-stage controlled study included 73 women with T2DM, which were divided into 2 groups depending on the functional state of the ovaries (reproductive and postmenopausal periods). In each group, subgroups of the Buryat and Russian populations were identified. The first group included 34 patients with T2DM of the reproductive period: 16 from the Buryat population and 18 from the Russian population. The second group consisted of 39 postmenopausal patients with T2DM: 17 from the Buryat population and 22 from the Russian population. The study of BMD in the lumbar spine (L1-L4), femoral neck (Neck), in the proximal femur (Total hip), trabecular bone score (TBS), serum osteocalcin (OC), N-terminal propeptide type 1 procollagen was carried out (P1NP), vitamin D 25 (OH), blood plasma type I collagen C-terminal telopeptide ( $\beta$-Cross laps) and ionized calcium (iCa).

RESULTS: In female patients with T2DM of the reproductive age of the Buryat population, an increase in both markers of osteosynthesis P1NP $(p=0.035), O C(p=0.047)$, and bone resorption $\beta$-Cross laps $(p=0.040)$ was found relative to the similar group of the Russian population. In women with T2DM in the postmenopausal period of the Buryat population, there was also an increase in P1NP ( $p=0.016)$, OC ( $p=0.048), \beta$-Cross laps $(p=0.020)$ compared with the group of postmenopausal women in the Russian population. Structural disorders, characterized by a decrease in TBS, were detected only in the postmenopausal period in female patients of the Buryat population compared to women in the Russian population $(p=0.029)$.

Comparative analysis among women with T2DM of the Buryat population, depending on the functional state of the ovaries, showed that activation of bone remodeling with an increase in P1NP $(p=0.019), O C(p=0.004)$ and $\beta$-Cross laps ( $p=0.004)$ is characteristic of postmenopausal women accompanied by a decrease in BMD Neck $(p=0.006), B M D$ Total hip $(p=0.003)$, BMD L1-L4 $(p=0.049)$ and TBS $(p=0.020)$ relative to female patients with T2DM in the reproductive period.

CONCLUSION: In women with T2DM in the Buryat population, both in the reproductive and postmenopausal periods, an increase in bone remodeling markers and BMD stability was found when compared with the corresponding groups of patients in the Russian population. The postmenopausal period was characterized by an additional decrease in TBS in patients with T2DM in the Buryat population relative to women in the Russian population.

KEYWORDS: diabetes mellitus; osteosynthesis; BMD; TBS; ethnic characteristics

\section{ОБОСНОВАНИЕ}

Гипергликемия при сахарном диабете (СД) способствует изменениям органов и тканей, в том числе и костной. По данным ряда авторов, у лиц пожилого возраста с СД 2 типа (СД2) наблюдается высокий риск остеопоротических переломов, что позволило выделить СД2 в качестве самостоятельного фактора риска остеопороза независимо от возраста, индекса массы тела (ИМТ) и минеральной плотности костной ткани (МПК) [1]. В клинических исследованиях показаны нарушения минерализации костной ткани у СД $[2,3]$. При СД2 рост МПК дает количественную оценку костной массы и не отражает прочность костной ткани $[4,5]$. I. De Liefde и соавт. считают, что использование параметров МПК становится не всегда информативным [6]. В последние годы для оценки микроархитектоники костной ткани шире используется трабекулярный костный индекс (ТКИ), относящийся к перспективным методам диагностики [5]. Продолжаются исследования, связанные с изучением патогенетических факторов, в том числе этнического характера, способных оказывать влияние на механизм формирования обменных процессов при диабете и его осложнениях. В этой связи являются актуальными работы о состоянии костной ткани и костного метаболизма у больных СД2 в разных этнических группах [7-9]. Работы по изучению структурно-метаболических особенностей костной ткани в бурятской популяции пока не проводились.

\section{ЦЕЛЬ ИССЛЕДОВАНИЯ}

Изучить показатели метаболизма костной ткани, микроархитектоники и МПК у пациенток с СД2 бурятской популяции.

\section{МАТЕРИАЛЫ И МЕТОДЫ}

Место и время проведения исследования

Место проведения. Исследование выполнено на базе клинико-диагностического центра ИГМАПО - филиала ФГБОУ ДПО РМАНПО Минздрава России, г. Иркутск.

Время исследования. Исследование проведено в период с 2016 по 2019 г.

Изучаемые популяции (одна или несколько)

В исследование были включены пациентки бурятской и русской популяций с диагнозом СД2.

Критериями исключения являлись: СД 1 типа и другие заболевания, приводящие к развитию вторичного остеопороза (ревматоидный артрит, хронические заболевания печени, онкологические заболевания, тиреотоксикоз, хроническая надпочечниковая недостаточность, гиперкортицизм), переломы в анамнезе, терапия глюкокортикоидами и антирезорбтивными препаратами.

Были сформированы две группы пациенток с СД2 в зависимости от функционального состояния яичников. Первую группу составили женщины, больные СД2, репродуктивного периода, которые были разделены на две подгруппы русской и бурятской популяций. Вторую группу сформировали пациентки с СД2 постменопаузального периода, которые также были разделены разделены на две подгруппы русской и бурятской популяций.

Способ формирования выборки из изучаемой популяции (или нескольких выборок из нескольких изучаемых популяций)

В исследовании применялся произвольный способ формирования выборок. 


\section{Дизайн исследования}

Проведено одноцентровое наблюдательное одномоментное двухвыборочное сравнительное исследование.

\section{Описание медицинского вмешательства (для}

интервенционных исследований)

Всех участниц обследовали по единому протоколу: после проведения анализа анамнестической информации, общеклинического обследования с занесением в анкеты, разработанные для исследования, проводился забор венозной крови утром натощак с 8 до 9 ч. МПК и ТКИ определяли с помощью двухэнергетической рентгеновской абсорбциометрии (DXA).

\section{Методы}

В исследование были включены пациентки бурятской и русской популяций с верифицированным диагнозом СД2 (BO3, 1999-2013). Национальная принадлежность определялась женщинами, считавшими себя и своих предков (3-4 поколения) бурятами или русскими. Функциональное состояние яичников оценивалось в соответствии с критериями STRAW+10. Критерии исключения определялись на врачебном приеме с учетом анализа медицинской документации.

Уровни С-концевого телопептида коллагена I типа ( $\beta$-Cross laps) и ионизированного кальция (iCa) плазмы крови, остеокальцина (ОК), N-терминального пропептида проколлагена 1-го типа (P1NP) и 25(OH) витамина D сыворотки крови определялись иммунохемилюминесцентным и ионоселективным методами (анализаторы Architect i2000, Cobas 601, Konelab PRIME 30, лаборатория «Инвитро»). Оценивали МПК, используя DXА проксимального отдела бедренной кости (Total hip), шейки бедра (Neck) и поясничных позвонков (L1-L4). На основе полученных данных был определен ТКИ поясничного отдела позвоночника (денситометр Prodigy, Lunar, GE Healhcare).

\section{Статистический анализ}

Размер выборки предварительно не рассчитывался. Методы статистического анализа данных: статистический ана- лиз осуществлялся с помощью пакета программ Statistica 10 (StatSoft Inc, США). Данные представлены в виде медианы (Ме) и квартилей (25 и 75 процентили, Q25-75). Сравнение независимых групп по количественным признакам осуществлялось непараметрическим методом с использованием U-критерия Манна-Уитни. Анализ корреляции переменных производился по методу Спирмена. Статистически значимыми считали различия при $\mathrm{p}<0,05$.

\section{Этическая экспертиза}

Протокол исследования одобрен на заседании Комитета по этике научных исследований ИГМАПО филиала ФГБОУ ДПО РМАНПО Минздрава России от 28 января 2016 г. (протокол №1). Всеми пациентами подписаны информированные согласия на участие в исследовании.

\section{РЕЗУЛЬТАТЫ}

В исследование включены 73 пациентки с СД2, которые в зависимости от функционального состояния яичников разделены на две группы: репродуктивного и постменопаузального периодов. В каждой группе были выделены подгруппы в зависимости от этнического статуса. Первая группа включала 34 женщины, больных СД2, репродуктивного периода, из них 16 пациенток бурятской популяции, медиана возраста 42,5 [41,5; 45,5] года, и 18 пациенток русской популяции, медиана возраста 44 [42; 47] года. Подгруппы женщин существенно не отличались по длительности СД2 и его осложнениям. Вторая группа сформирована из 39 женщин, больных СД2, постменопаузального периода, из них 17 пациенток бурятской популяции, медиана возраста 59 [57; 61] лет, и 22 пациентки русской популяции, медиана возраста 57,5 [55; 62] года. Подгруппы женщин также существенно не отличались по длительности СД и его осложнениям.

Установлено, что у пациенток с СД2 репродуктивного периода бурятской популяции (табл. 1) наблюдалось

Таблица 1. Результаты исследования показателей двухэнергетической рентгеновской абсорбциометрии и маркеров костного метаболизма у женщин репродуктивного периода (результаты представлены в виде Ме [Q1; Q3])

\begin{tabular}{|c|c|c|c|}
\hline Показатель & $\begin{array}{l}\text { Бурятская популяция } \\
(n=16)\end{array}$ & $\begin{array}{l}\text { Русская популяция } \\
(\mathrm{n}=18)\end{array}$ & p-value \\
\hline Возраст, лет & $42,5[41,5 ; 45,5]$ & $44[42 ; 47]$ & 0,321 \\
\hline 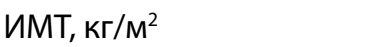 & $32,9[30,5 ; 35,9]$ & $32,5[30,9 ; 34,7]$ & 0,783 \\
\hline $\mathrm{HbA}_{1 c^{\prime}} \%$ & $7,5[6,8 ; 7,7]$ & $7,2[7,0 ; 7,5]$ & 0,406 \\
\hline 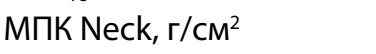 & $1,000[0,951 ; 1,094]$ & $1,037[0,945 ; 1,104]$ & 0,969 \\
\hline 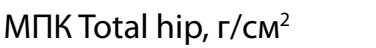 & $1,144[1,075 ; 1,236]$ & $1,170[1,117 ; 1,264]$ & 0,406 \\
\hline MПK L1-L4, г/CM ${ }^{2}$ & $1,203[1,077 ; 1,347]$ & $1,258[1,176 ; 1,324]$ & 0,418 \\
\hline ТКИ & $1,338[1,262 ; 1,379]$ & $1,324[1,206 ; 1,411]$ & 0,797 \\
\hline$\beta$-Cross laps, нг/мл & $0,228[0,194 ; 0,367]$ & $0,160[0,114 ; 0,244]$ & 0,040 \\
\hline ОК, нг/мл & $12,0[8,0 ; 15,5]$ & $9,5[7,0 ; 11,0]$ & 0,047 \\
\hline P1NP, нг/мл & $30,5[24,5 ; 38,7]$ & $24,2[17,3 ; 31,8]$ & 0,035 \\
\hline 25(OH) витамин D, нг/мл & $18,0[14,0 ; 24]$ & $25,0[20,0 ; 27,0]$ & 0,084 \\
\hline іСа, ммоль/л & $1,11[1,09 ; 1,14]$ & $1,12[1,10 ; 1,15]$ & 0,499 \\
\hline
\end{tabular}

Примечание. ИМТ — индекс массы тела; OK — остеокальцин; P1NP — N-терминальный пропептид проколлагена 1-го типа; $\beta$-Сross laрs - C-концевые телопептиды коллагена I типа; МПК - минеральная плотность костной ткани; Neck — шейка бедра; Тоtal hip — проксимальный отдел бедренной кости; L1-L4 - поясничный отдел позвоночника; TKИ - трабекулярный костный индекс, iCa - ионизированный кальций. 
Таблица 2. Результаты исследования показателей двухэнергетической рентгеновской абсорбциометрии и маркеров костного метаболизма у женщин в постменопаузальном периоде (результаты представлены в виде Me [Q1; Q3])

\begin{tabular}{|c|c|c|c|}
\hline Показатель & $\begin{array}{l}\text { Бурятская популяция } \\
(\mathrm{n}=17)\end{array}$ & $\begin{array}{l}\text { Русская популяция } \\
\text { (n=22) }\end{array}$ & p-value \\
\hline Возраст, лет & $59[57 ; 61]$ & $57,5[55 ; 62]$ & 0,712 \\
\hline ИМТ, кг/м² & $30,1[28,5 ; 31,6]$ & $30,9[27,4 ; 34,1]$ & 0,777 \\
\hline $\mathrm{HbA}_{1 c^{\prime}} \%$ & $7,5[7,0 ; 7,6]$ & $7,5[7,1 ; 7,8]$ & 0,434 \\
\hline МПК Neck, г/см² & $0,875[0,759 ; 0,971]$ & $0,940[0,886 ; 0,988]$ & 0,179 \\
\hline 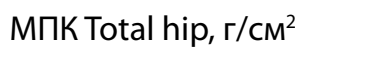 & $1,002[0,913 ; 1,075]$ & $1,024[0,949 ; 1,072]$ & 0,453 \\
\hline 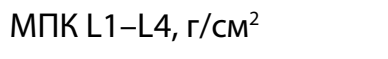 & $1,072[1,015 ; 1,191]$ & $1,099[1,025 ; 1,185]$ & 0,745 \\
\hline FRAX, \% & $10,5[7,4 ; 12,0]$ & $7,9[7,3 ; 9,9]$ & 0,251 \\
\hline ТКИ & $1,191[1,156 ; 1,306]$ & $1,314[1,234 ; 1,367]$ & 0,029 \\
\hline$\beta$-Cross laps, нг/мл & $0,450[0,284 ; 0,560]$ & $0,254[0,158 ; 0,409]$ & 0,020 \\
\hline ОК, нг/мл & $16,0[14,0 ; 22,0]$ & $14,0[12,0 ; 17,0]$ & 0,048 \\
\hline P1NP, нг/мл & $39,2[32,8 ; 47,4]$ & $27,5[22,6 ; 40,8]$ & 0,016 \\
\hline 25(OH) витамин D, нг/мл & $18,0[15,0 ; 21,0]$ & $20,0[17,0 ; 27,0]$ & 0,052 \\
\hline іСа, ммоль/л & $1,13[1,10 ; 1,16]$ & $1,13[1,10 ; 1,17]$ & 0,649 \\
\hline
\end{tabular}

Примечание. ИМТ - индекс массы тела, ОK - остеокальцин, P1NP - N-терминальный пропептид проколлагена 1-го типа, $\beta$-Сross laps - C-концевые телопептиды коллагена I типа, МПК - минеральная плотность костной ткани, Neck - шейка бедра, Тоtal hip - проксимальный отдел бедренной кости, L1-L4 - поясничный отдел позвоночника, ТKИ - трабекулярный костный индекс, іСа - ионизированный кальций.

повышение показателей P1NP $(p=0,035)$, OK $(p=0,047)$ и $\beta$-Cross laps $(p=0,040)$ относительно пациенток с СД2 репродуктивного периода русской популяции. Показатели МПК и ТКИ женщин, больных СД2, бурятской популяции репродуктивного периода были на уровне соответствующих показателей пациенток с СД2 русской популяции репродуктивного периода ( $p>0,05)$.

У пациенток с СД2 постменопаузального периода бурятской популяции процессы ремоделирования костной ткани характеризовались увеличением уровней P1NP $(p=0,016)$, OK $(p=0,048)$ и $\beta$-Cross laps $(p=0,020)$ относительно женщин с СД2 периода постменопаузы русской популяции (табл. 2). Обращает внимание снижение значений ТКИ у пациенток с СД2 бурятской популяции сравнительно с женщинами, больными СД2, в периоде постменопаузы русской популяции на фоне отсутствия различий в показателях МПК $(p=0,029)$.

Проведенный сравнительный анализ состояния костной ткани в зависимости от функциональной активности яичников показал, что для женщин бурятской популяции с СД2 периода постменопаузы характерно повышение маркеров остеосинтеза (OK $(p=0,004)$ и P1NP $(p=0,019))$ с увеличением маркера остеорезорбции $(\beta$-Cross laps $(p=0,004))$ на фоне снижения ТКИ $(p=0,020)$ относительно соответствующих данных пациенток с СД2 репродуктивного возраста (табл. 1). У пациенток с СД2 русской популяции состояние постменопаузы сопровождалось только повышением концентрации ОК $(p=0,001)$ и $\beta$-Cross laps $(p=0,013)$ на фоне отсутствия различий показателей ТКИ $(p=0,745)$ относительно пациенток с СД2 репродуктивного периода русской популяции.
У пациенток с диабетом бурятской популяции постменопаузальный период характеризовался снижением показателей МПК Neck $(p=0,006)$, MПK Total hip $(p=0,003)$ и MПK L1-L4 ( $p=0,049)$ по сравнению с аналогичными показателями репродуктивного периода. У женщин с СД2 русской популяции в постменопаузальном периоде также наблюдалось снижение МПК Neck $(p=0,024)$, MПK Total hip $(p=0,002)$ и MПK L1-L4 ( $p=0,001)$ относительно соответствующих показателей пациенток репродуктивного периода.

Корреляционный анализ установил положительные взаимосвязи 25(OH) витамина D c OK $(p=0,71)$, P1NP $(p=0,56)$ у женщин, больных СД2, репродуктивного периода бурятской популяции. Кроме этого, для женщин-буряток с диабетом репродуктивного периода была характерна корреляционная взаимосвязь ТКИ и MПK L1-L4 $(p=0,59)$, ТКИ от ИМТ $(p=0,53)$ и отрицательная взаимосвязь ТКИ и Р1NP $(p=-0,56)$.

Выраженная тенденция к снижению концентрации 25(OH) витамина D отмечена у пациенток с СД2 в постменопаузальном периоде бурятской популяции относительно женщин с диабетом периода постменопаузы русской популяции $(p=0,052)$. Менее выраженная тенденция к снижению 25(OH) витамина D наблюдалась у женщин-буряток с диабетом репродуктивного периода относительно аналогичной подгруппы женщин русской популяции (табл. 1 и 2). Сравнительный анализ показал, что особой стабильностью обладает іСа, уровни которого не отличались между группами и подгруппами женщин ( $p>0,05)$.

Во время исследования нежелательных явлений не отмечено. 


\section{ОБСУЖДЕНИЕ}

\section{Репрезентативность выборок}

Набор участников исследования проводился на базе клинико-диагностическом центра ИГМАПО - филиала ФГБОУ ДПО РМАНПО Минздрава России (г. Иркутск).

\section{Сопоставление с другими публикациями}

Низкие значения МПК у пациенток с СД2 в постменопаузальном периоде по сравнению с репродуктивным возрастом согласуются с данными других исследователей, которые показали увеличение темпов снижения МПК уженщин в возрасте старше 40-45 лет, что обусловлено дефицитом эстрогенов в период постменопаузы. Известно, что распространенность переломов коррелирует с низкими показателями МПК поясничного отдела позвоночника и проксимальных отделов бедренных костей $[3,5]$. Наши данные также показали снижение МПК поясничного отдела позвоночника, шейки бедра, проксимального отдела бедренной кости у пациенток с СД2 постменопаузального периода относительно женщин репродуктивного периода как русской, так и бурятской популяций. Нами не выявлено разницы в МПК при сравнении подгрупп женщин бурятской и русской популяций как репродуктивного периода, так и постменопаузального.

У женщин с СД2 в постменопаузе бурятской популяции установлены низкие значения ТКИ относительно пациенток с диабетом в постменопаузе русской популяции (табл. 2). Проведенный нами сравнительный анализ показал снижение ТКИ у женщин в постменопаузе относительно репродуктивного периода бурятской популяции. Как известно, снижение ТКИ может свидетельствовать о нарушении микроархитектоники костной ткани с высоким риском низкотравматичных переломов. Вместе с тем ТКИ отражает структуру трабекулярной ткани поясничных позвонков и способен более точно прогнозировать риск низкотравматических переломов при диабете [10]. Согласно исследованиям, к основным факторам нарушений костной ткани при СД2 относят деградацию микроархитектоники со снижением качественных характеристик, а не депрессию МПК [11, 12].

Нами показано, что в бурятской популяции у женщин с СД2 в постменопаузе наблюдается одновременное увеличение остеорезорбции (повышение $\beta$-Cross laps) и остеосинтеза (повышение P1NP, ОК) относительно женщин репродуктивного периода (табл. 1, 2). В свою очередь, в русской популяции у пациенток с СД2 в постменопаузальном периоде выявлено одновременное повышение OK и $\beta$-Cross laps по сравнению с женщинами репродуктивного периода. По мнению ряда авторов, процессы костного ремоделирования оказывают непосредственное влияние на состояние МПК [13-15]. Наши данные согласуются с результатами авторов, продемонстрировавших в постменопаузальном периоде параллельное повышение маркеров остеорезорбции и остеосинтеза, что ассоциируется с потерей костной массы $[16,17]$.

Проведенное исследование показало, что у женщин с СД2 как репродуктивного, так и постменопаузального периодов бурятской популяции зафиксировано значимое повышение P1NP, OK и $\beta$-Cross laps по сравнению с показателями аналогичных подгрупп пациенток с СД2 русской популяции, что свидетельствует об активации как остеорезорбции, так и остеосинтеза. Подобный метаболизм костной ткани в репродуктивном периоде у женщин с СД2 бурятской популяции способен приводить к негативным изменениям в костях с повышением риска переломов.

У женщин бурятской популяции до и после менопаузы отмечалась тенденция к снижению уровней 25(OH) витамина D относительно соответствующих групп женщин русской популяции (табл. 1 и 2), что обусловлено более темным тоном кожи, а также, возможно, и эпигенетическими особенностями в метаболизме витамина D. Установленная положительная корреляционная связь 25(OH) витамина D и маркеров костного синтеза у женщин, больных СД2, репродуктивного периода бурятской популяции способна оказывать влияние на процессы костного ремоделирования.

\section{Клиническая значимость результатов}

Оптимальный выбор диагностических мероприятий способствует оценке риска структурно-метаболических нарушений костной ткани у женщин, больных СД2, репродуктивного и постменопаузального периодов разных этнических групп и подтверждает роль своевременной профилактики с коррекцией наиболее значимых «управляемых» факторов риска. Полученные результаты могут быть использованы при составлении программ лечебно-профилактического характера в регионах с неоднородным по национальному составу населением.

\section{Ограничения исследования}

Одномоментный дизайн не позволяет судить о причинно-следственных взаимосвязях между признаками. Объем выборки требует осторожности при интерпретации полученных данных.

\section{Направления дальнейших исследований}

Дальнейшее изучение этнических аспектов состояния костной ткани у больных СД2 с оценкой факторов риска заключается в необходимости планирования проведения дальнейших исследований с формированием расширенных групп.

\section{ЗАКЛЮЧЕНИЕ}

Проведенное исследование показало, что у женщин, больных СД2, бурятской популяции независимо от состояния функции яичников наблюдается повышение маркеров костного ремоделирования (P1NP, OK и $\beta$-Cross laps) относительно пациенток с СД2 русской популяции. МПК и ТКИ у женщин, больных СД2, репродуктивного периода бурятской популяции соответствовали показателям русской популяции. Показатели МПК у женщин с диабетом постменопаузального периода бурятской и русской популяций не отличались, однако у пациенток бурятской популяции наблюдалась депрессия ТКИ.

Таким образом, для женщин, больных СД2, репродуктивного периода бурятской популяции характерно только ускорение процессов ремоделирования костной ткани, а в периоде постменопаузы дополнительно определяются структурные изменения. Есть основания считать, что выявленные структурно-метаболические изменения костной ткани носят этнический характер и могут способствовать негативным последствиям, в том числе повышению риска переломов у женщин бурятской популяции. 


\section{ДОПОЛНИТЕЛЬНАЯ ИНФОРМАЦИЯ}

Источники финансирования. Исследование проведено за счет бюджетных средств организации.

Конфликт интересов. Авторы декларируют отсутствие явных и потенциальных конфликтов интересов, связанных с содержанием настоящей статьи.

Участие авторов. Бардымова Т.П. - концепция и дизайн исследования, анализ данных, написание текста; Шестакова М.В. - концепция и идея; Сандаков Я.П. - дизайн исследования, анализ данных; Мистяков М.В. - анализ литературы, сбор клинического материала, статистическая обработка результатов, написание основного текста; Березина М.В. - анализ литературы, написание основного текста. Все авторы одобрили финальную версию статьи перед публикацией, выразили согласие нести ответственность за все аспекты работы, подразумевающую надлежащее изучение и решение вопросов, связанных с точностью или добросовестностью любой части работы.

\section{СПИСОК ЛИТЕРАТУРЫ | REFERENCES}

1. Ялочкина Т.О., Белая Ж.Е., Рожинская Л.Я., и др. Переломы костей при сахарном диабете 2 типа: распространенность и факторы риска // Сахарный диабет. - 2016. - Т. 19. - №5. - C. 359-365. [Yalochkina TO, Belaya JE, Rozhinskaya LY, et al. Bone fractures in patients with type 2 diabetes mellitus: prevalence and risk factors. Diabetes mellitus. 2016; 19(5):359-365. (In Russ.)]. doi: https://doi.org/10.14341/dm7796

2. Ross PD, Knowlton W. Rapid bone loss is associated with increased levels of biochemical markers. J Bone Miner Res. 1998;13(2):297-302. doi:10.1359/jbmr.1998.13.2.297

3. Schousboe JT, Bauer DC, Nyman JA, et al. Potential for bone turnover markers to cost-effectively identify and select postmenopausal osteopenic women at high risk of fracture for bisphosphonate therapy. Osteoporos Int. 2007;18(2):201-210. doi: https://doi.org/10.1007/s00198-006-0218-7

4. Vestergaard P. Discrepancies in bone mineral density and fracture risk in patients with type 1 and type 2 diabetes-a meta-analysis. Osteoporos Int. 2007;18(4):427-444. doi: https://doi.org/10.1007/s00198-006-0253-4

5. Дедов И.И., Мельниченко Г.А., Белая Ж.Е., и др. Остеопороз от редкого симптома эндокринных болезней до безмолвной эпидемии XX-XXI века // Проблемы Эндокринологии. - 2011. T. 57. — №1 — C. 35-45. [Dedov II, Mel'nichenko GA, Belaia ZE, et al. Osteoporosis: from a rare symptom of endocrine diseases to the tacit epidemic of XX-XXI centuries. Problems of Endocrinology. 2011;57(1):35-45. (In Russ.)]. doi: https://doi.org/10.14341/probl201157135-45

6. de Liefde II, van der Klift M, de Laet CE, et al. Bone mineral density and fracture risk in type-2 diabetes mellitus: the Rotterdam Study. Osteoporos Int. 2005;16(12):1713-1720. doi: https://doi.org/10.1007/s00198-005-1909-1

7. Yan L, Crabtree NJ, Reeve J, et al. Does hip strength analysis explain the lower incidence of hip fracture in the People's Republic of China? Bone. 2004;34(3):584-588. doi: https://doi.org/10.1016/j.bone.2003.12.005
8. Barrett-Connor E, Siris ES, Wehren LE, et al. Osteoporosis and fracture risk in women of different ethnic groups. J Bone Miner Res. 2005;20(2):185-194. doi: https://doi.org/10.1359/JBMR.041007

9. Ballane G, Cauley JA, Luckey MM, El-Hajj Fuleihan G. Worldwide prevalence and incidence of osteoporotic vertebral fractures. Osteoporos Int. 2017;28(5):1531-1542. doi: https://doi.org/10.1007/s00198-017-3909-3

10. Leslie WD, Aubry-Rozier B, Lamy O, Hans D. TBS (Trabecular Bone Score) and Diabetes-Related Fracture Risk. J Clin Endocr Metab. 2013;98(2):602-609. doi: 10.1210/jc.2012-3118

11. Jackuliak P, Payer J. Corrigendum to «Osteoporosis, Fractures, and Diabetes». Int J Endocrinol. 2017;2017:2846080. doi: https://doi.org/10.1155/2017/2846080

12. Ferrari SL, Abrahamsen B, Napoli N, et al. Diagnosis and management of bone fragility in diabetes: an emerging challenge. Osteoporos Int. 2018;29(12):2585-2596. doi: https://doi.org/10.1007/s00198-018-4650-2

13. Brandi ML. Microarchitecture, the key to bone quality. Rheumatology. 2009;48(Suppl 4):iv3-8 doi: https://doi.org/10.1093/rheumatology/kep273

14. Ulivieri FM, Silva BC, Sardanelli F, et al. Utility of the trabecular bone score (TBS) in secondary osteoporosis. Endocrine. 2014;47(2):435-448. doi: https://doi.org/10.1007/s12020-014-0280-4

15. Link TM, Majumdar S. Current diagnostic techniques in the evaluation of bone architecture. Curr Osteoporos Rep. 2004;2(2):47-52. doi: https://doi.org/10.1007/s11914-004-0003-5

16. Ross PD, Knowlton W. Rapid bone loss is associated with increased levels of biochemical markers. J Bone Miner Res. 1998;13(2):297-302. doi: https://doi.org/10.1359/jbmr.1998.13.2.297

17. Garnero P, Sornay-Rendu E, Duboeuf F, Delmas PD. Markers of bone turnover predict postmenopausal forearm bone loss over 4 years: the OFELY study. J Bone Miner Res. 1999; 14(9):1614-1621. doi: https://doi.org/10.1359/jbmr.1999.14.9.1614

\section{ИНФОРМАЦИЯ ОБ АВТОРАХ [AUTHORS INFO]}

*Бардымова Татьяна Прокопьевна, д.м.н., профессор [Tatiana P. Bardymova, MD, PhD, Professor]; aдpec: 664049, Российская Федерация, г. Иркутск, мкр. Юбилейный, 100 [address: 664049, Russia, Irkutsk, mkr. Jubilejny, 100]; ORCID: https://orcid.org/0000-0003-4241-2217; eLibrary SPIN: 6151-1430; e-mail: tpbardymova@mail.ru

Шестакова Марина Владимировна, д.м.Н., профессор, академик РАH [Marina V. Shestakova, MD, PhD, Professor]; ORCID: https://orcid.org/0000-0002-5057-127X; eLibrary SPIN: 7584-7015, e-mail: shestakova.mv@gmail.com

Сандаков Яков Павлович, д.м.н. [Yakov P. Sandakov]; ORCID: https://orcid.org/0000-0002-3976-9509; eLibrary SPIN: 8133-1812; e-mail: sand_2004@mail.ru

Мистяков Максим Викторович [Maksim V. Mistiakov]; ORCID: https://orcid.org/0000-0002-4675-3396;

eLibrary SPIN: 6430-3185; e-mail: mrdrx@mail.ru

Березина Марина Витальевна [Marina V. Berezina]; ORCID: https://orcid.org/0000-0002-4000-9566; eLibrary SPIN: 8697-1264; e-mail: berezin_e_v@bk.ru

\section{ЦИТИРОВАТЬ:}

Бардымова Т.П., Шестакова М.В., Сандаков Я.П., Мистяков М.В., Березина М.В. Этнические особенности ремоделирования костной ткани у пациенток с сахарным диабетом 2 типа // Сахарный диабет. - 2021. — Т. 24. - №5. — С. $427-432$. doi: https://doi.org/10.14341/DM12795

\section{TO CITE THIS ARTICLE:}

Bardymova TP, Shestakova MV, Sandikov YaP, Mistiakov MV, Berezina MV. Ethnic characteristics of bone remodeling in female patients with type 2 diabetes mellitus. Diabetes Mellitus. 2021;24(5):427-432. doi: https://doi.org/10.14341/DM12795 